\title{
PARETO OPTIMALITY AND EQUILIBRIUM IN AN INSURANCE MARKET
}

\author{
BY
}

\section{A.Y. Golubin}

\begin{abstract}
The concept of economic equilibrium under uncertainty is applied to a model of insurance market where, in distinction to the classic Borch's model of a reinsurance market, risk exchanges are allowed between the insurer and each insured only, not among insureds themselves. Conditions characterizing an equilibrium are found. A variant of the conditions, based on the Pareto optimality notion and involving risk aversion functions of the agents, is derived. An existence theorem is proved. Computation of the market premiums and optimal indemnities is illustrated by an example with exponential utility functions.
\end{abstract}

\section{KEYWORDS}

Insurance, premium, indemnity function, Pareto optimality, equilibrium.

\section{INTRODUCTION}

We investigate the problem of finding an equilibrium for a model of an insurance market in which each potential policyholder (insured) shares his initial risk with the insurer (paying a premium to the insurer) without possibility of risk exchange with the other insureds.

The notion of Walrasian equilibrium borrowed from mathematical economics has been applied to insurance models since Borch's works $(1960,1962)$. This concept implies that contracts made in the market are Pareto-optimal and, which is of importance, premiums of the contracts are determined by the market conditions as a whole, not only by covered risks. Borch's theorem on Pareto-optimal risk exchanges in the model of a reinsurance market was extended to a constrained case in Gerber (1978), an overview of applications of the Borch's theorem can be found in Lemaire (1990). A characterization of equilibrium in the reinsurance market was developed in Buhlmann $(1980,1984)$ and later in Aase $(1993,2002)$ where risk allocation problems in financial markets were also studied. Actually, a particular case of the model suggested in the paper, where risk is allocated between insurer and the only insured $(n=1)$, 
has been studied in the frame of the Borch's model of reinsurance market by several authors, e.g., Lemaire (1990), Aase (1993, 2002). The presented work is expected to be consistent with the known results for the case $n=1$.

Our setting differs from the classical Borch's model of a reinsurance market by the following: in the suggested model, each insured is independent of the other insureds in the sense that he concludes a treaty on risk exchange with the insurer only, isolated from the other insureds. In particular, the agents cannot hand in all their initial risks to a pool and then distribute shares of the summary risk back, as suggested by the optimal risk sharing rules in the reinsurance market model. Such independence (or separation) of insureds is justified by that we regard them as individual buyers of direct insurance, not as (re)insurance companies that might trade risks among themselves. It is worth noting that the model is not within the monopoly theory, according to which the monopolist (insurer) maximizes his utility constrained by that the insured's utility equals indifference value. However, the insured may just refuse the insurance contract, not losing anything of his utility, while the related decrease in insurer's utility is then quite perceptible. In this sense, the monopoly solution is not stable. The variation of the Borch's model suggested in the paper is free of such a disadvantage (and, by the way, is "more fair" for the insureds): in the equilibrium each agent has his maximal utility under equilibrium prices and has no desire to change the decision.

The main purpose of the paper is to give necessary and sufficient conditions for determining an equilibrium and then to compare them with the characterization of equilibrium in the classical model of a reinsurance market. The equilibrium notion employed in the present work substantiates a choice of a single contract among the family (generally uncountable) of Pareto-optimal contracts. The paper uses results in Golubin (2005) on description of Pareto optima in the insurance market.

The article is organized as follows. In section 2 the model of an insurance market under investigation is presented. Section 3 gives a characterization of an equilibrium in the market. Section 4 deals with Pareto-optimal contracts and provides two variants of necessary and sufficient conditions for Pareto optimality in the model. In section 5 we derive an equilibrium condition based on the Pareto optimality characterization and involving the risk aversion (tolerance) functions of the agents. We end the exposition with a discussion (section 6) of the equilibrium existence problem.

\section{THE MARKET MODEL}

We study a market consisting of $n+1$ agents: an insurer and a group of $n$ insureds. The initial insureds' risks (losses) $X_{j}, j=1, \ldots, n$ are nonnegative independent stochastic variables defined on the same probability space $(\Omega, \mathcal{F}, P)$. The sigma-algebra of events $\mathcal{F}=\sigma(X)$ is generated by the initial risks portfolio $X=\left(X_{1}, \ldots, X_{n}\right)$. It means that the uncertainty in the model is completely 
described by the initial risks $X_{j}$. The distribution function of $X_{j}$ is denoted by $F_{j}(x) \stackrel{\text { def }}{=} P\left\{X_{j} \leq x\right\}$.

The agents' preferences are represented by their utility functions $u_{i}(x)$ such that $u_{i}^{\prime}(x)>0$ and $u_{i}^{\prime \prime}(x)<0$ for all $i=0, \ldots, n$.

The insurer and an insured negotiate to conclude a treaty on risk exchange between them. We suppose that any coalitions within the group of insureds are not allowed, so each insured stands as a separate decision maker in bargaining with the insurer. A resulting risk exchange is identified with a set of Borelmeasurable functions $I=\left(I_{1}, \ldots, I_{n}\right)$ called indemnity functions or policies, defined on $[0, \infty)$, and satisfying the standard constraints $0 \leq I_{j}(x) \leq x$ for $j=1, \ldots, n$. This means that an indemnity payment $I_{j}\left(X_{j}\right)$ to the $j$-th insured is always nonnegative and not greater than the loss size $X_{j}$. Like in the model of a reinsurance market studied in Borch (1962) and Buhlmann $(1980,1984)$, we introduce a price functional that assigns the premium $H[Y]$ for a risk $Y$ of an insured as

$$
H[Y]=E[\Phi Y]
$$

Here $\Phi$ is a positive stochastic variable defined on $(\Omega, \mathcal{F}, P)$ and therefore it can be represented as an appropriate Borel-measurable function (see, e.g., Tucker (1967)), $\Phi=\phi\left(X_{1}, \ldots, X_{n}\right)$. The price of a constant risk must be the same constant, so we assume $E \Phi=1$. Following Buhlmann, we will call $\Phi$ a market price density. An explanation of the term is that one can interpret $H[Y]$ as an expectation of $Y$ with respect to a distorted probability measure, $E_{Q} Y=E[\Phi Y]$. Here $\Phi$ is the Radon-Nikodym derivative of measure $Q$ with respect to the original measure $P$. The linearity of the price functional allows for eliminating a kind of arbitrage possibilities: Let $Y_{1}$ be a share of an insured's risk $X_{j}$ and $Y_{2}=X_{j}-Y_{1}$ be the rest of the risk. The insurer may, first, insure the entire risk $Y_{1}+Y_{2}$ or, second, do it by parts: to insure the share $Y_{1}$ and, immediately after that, $Y_{2}$. If, say, $H\left[Y_{1}+Y_{2}\right]<H\left[Y_{1}\right]+H\left[Y_{2}\right]$ then in the second case the insurer raises the premium increment from nothing. Such a situation should not be possible in any consistent model of the insurance market. Thus the price functional $H$ must be linear (with respect to risks of each insured).

The expected utilities of final capitals of the insurer and $j$-th insured are $J_{0}[I] \stackrel{\text { def }}{=} E u_{0}\left(w_{0}+\sum_{s=1}^{n}\left(P_{s}-I_{s}\left(X_{s}\right)\right)\right)$ and $J_{j}[I] \stackrel{\text { def }}{=} E u_{j}\left(w_{j}-P_{j}-X_{j}+I_{j}\left(X_{j}\right)\right), j=1$, $\ldots, n$. Here $P_{j}=E\left[\Phi I_{j}\left(X_{j}\right)\right]$ is the premium paid by $j$-th insured to the insurer, $w_{i}, i=0, \ldots, n$, denote initial nonstochastic capitals of the agents. In the sequel, all the expectations, e.g. the premiums $E\left[\Phi I_{j}\left(X_{j}\right)\right]$ and expected utilities, are assumed to be finite.

It is essential to note that $E\left[\Phi I_{j}\left(X_{j}\right)\right]=E\left[\Psi_{j} I_{j}\left(X_{j}\right)\right]$, where

$$
\Psi_{j}=E\left[\Phi \mid X_{j}\right]
$$

is the conditional expectation of $\Phi$ with respect to a sigma-algebra $\sigma\left(X_{j}\right)$ generated by $X_{j}$. Thus, to define all the premiums $P_{j}=E\left[\Psi_{j} I_{j}\left(X_{j}\right)\right]$ entering the 
functionals $J_{i}$, it suffices to define, instead of $\Phi$, the stochastic variables $\Psi_{j}$, $j=1, \ldots, n$. By the construction, each $\Psi_{j}$ is positive and such that $E \Psi_{j}=1$. Since the conditional expectation is $\sigma\left(X_{j}\right)$-measurable, it can also be represented as $\Psi_{j}=\psi_{j}\left(X_{j}\right)$ a.s., where $\psi_{j}(x)$ is some Borel-measurable function. More exactly, $\psi_{j}\left(X_{j}\right)$ is a result of taking expectation of $\Phi=\phi\left(X_{1}, \ldots, X_{n}\right)$ with respect to $X_{1}, \ldots, X_{j-1}, X_{j+1}, \ldots, X_{n}$. In the sequel we will call $\Psi_{j}$ the $j$-th price density.

Define the notion of equilibrium in the insurance market as follows: Price densities $\Psi=\left(\Psi_{1}, \ldots, \Psi_{n}\right)$ and policies $I=\left(I_{1}, \ldots, I_{n}\right)$ constitute an equilibrium if $I_{j}, j=1, \ldots, n$, solve the problems

$$
\max _{I} J_{i}[I], i=0, \ldots, n .
$$

The problem of finding an equilibrium is then to determine from the agents' preferences and distributions $F_{j}(x)$ of the insureds' risks: (a) the price functions $\psi_{j}(x)$; (b) the policies $I_{j}(x)$ solving problems (3) in which the premiums depend on policies as $P_{j}=E\left[\psi_{j}\left(X_{j}\right) I_{j}\left(X_{j}\right)\right]$.

After obtaining the price densities $\Psi_{j}=\psi_{j}\left(X_{j}\right)$, one may construct the market price density as

$$
\Phi=\prod_{k=1}^{n} \Psi_{k}
$$

Indeed, taking into account (2), independence of all $\Psi_{k}$, and the fact that any priced risk $Y=I_{j}\left(X_{j}\right)$ depends on variable $X_{j}$ only, we have that the premium calculated via "initial" market price density, $P_{j}=E[\Phi Y]$, coincides with $P_{j}=$ $E\left\{\prod_{k=1}^{n} \Psi_{k} \cdot Y\right\}=E\left\{\prod_{k \neq j}^{n} \Psi_{k}\right\} E\left[\Psi_{j} Y\right]=E\left[\Psi_{j} Y\right]$.

Remark in conclusion that the only difference between the presented definition of equilibrium and the equilibrium in the reinsurance market model (see, e.g., Buhlmann (1984), Aase (1993)) follows the difference in definitions of an admissible risk exchange: Maxima in (3) are taken over indemnities $I_{j}\left(X_{j}\right)$ each depending on $j$-th risk only and satisfying the constraints $0 \leq I_{j}\left(X_{j}\right) \leq X_{j}$ a.s., $j=1, \ldots, n$. In the corresponding $(n+1)$-agent Borch's model, where the initial risks are $\left(0, X_{1}, \ldots, X_{n}\right)$, all risk exchanges $Z_{i}$ that satisfy market clearing condition $\sum_{i=0}^{n} Z_{i}=\sum_{j=1}^{n} X_{i}$ are admissible in maximization. The market clearing condition is evidently met also in our setting, where the $n+1$ agents only trade among themselves. Like in Buhlmann (1984), due to linearity of the price functional $H[Y]$ the price of the wealth after exchange is equal to the price of the initial wealth (the budget constraints) for all the agents. So problems (3) are defined over a set of risk exchanges which is narrower than that in the Borch's model of a reinsurance market.

\section{EQUILIBRIUM CHARACTERIZATION}

The next theorem presents necessary and sufficient conditions for determination of an equilibrium. For convenience, we will use designations $A=w_{0}+$ 
$\sum_{s=1}^{n}\left(P_{s}-I_{s}\left(X_{s}\right)\right)$ and $B_{j}=w_{j}-P_{j}-X_{j}+I_{j}\left(X_{j}\right)$ for the final capitals of the insurer and insureds respectively.

Theorem 1. Collections $\Psi=\left(\Psi_{1}, \ldots, \Psi_{n}\right)$ of price densities and $I=\left(I_{1}, \ldots, I_{n}\right)$ of policies are an equilibrium if and only if

$$
\begin{aligned}
& E\left[u_{0}^{\prime}(A) \mid X_{j}\right]=\Psi_{j} E u_{0}^{\prime}(A) \text { a.s. } \\
& u_{j}^{\prime}\left(B_{j}\right)=\Psi_{j} E u_{j}^{\prime}\left(B_{j}\right) \text { a.s. for } j=1, \ldots, n .
\end{aligned}
$$

Proof. Denote by $I$ a maximizer in problems (3). Fix any (admissible) indemnity rule $L$ and consider the policies $I_{j}(x)+\lambda \Delta_{j}(x)$, where $\lambda \in[0,1]$ is a parameter and $\Delta_{j}(x)=L_{j}(x)-I_{j}(x), j=1, \ldots, n$. By optimality of $I$, the point $\lambda=0$ maximizes all the functions $J_{i}(\lambda)=J_{i}[I+\lambda \Delta]$ on the interval $[0,1]$. Due to concavity of $J_{i}[L]$, the fulfillment of the inequalities

$$
\left.J_{i}^{\prime}(\lambda)\right|_{\lambda=0} \leq 0
$$

for any indemnity rule $L$ and for all $i=0, \ldots, n$ is necessary and sufficient for optimality in (3). After differentiating each $J_{i}(\lambda)$, it can be easily seen that (7) is equivalent to:

$$
\begin{aligned}
& E\left\{\left[-E\left[u_{0}^{\prime}(A) \mid X_{j}\right]+\psi_{j}\left(X_{j}\right) E u_{0}^{\prime}(A)\right] \Delta_{j}\left(X_{j}\right)\right\} \leq 0, \\
& E\left\{\left[u_{j}^{\prime}\left(B_{j}\right)-\psi_{j}\left(X_{j}\right) E u_{j}^{\prime}\left(B_{j}\right)\right] \Delta_{j}\left(X_{j}\right)\right\} \leq 0
\end{aligned}
$$

for all $j=1, \ldots, n$. This means that policies $I_{j}$ solve (3) if and only if they maximize the integrals

$$
\begin{aligned}
& \int_{0}^{\infty}\left[\psi_{j}(x)-q_{j}(x)\right] L_{j}(x) d F_{j}(x) \text { subject to } 0 \leq L_{j}(x) \leq x, \\
& \int_{0}^{\infty}\left[r_{j}(x)-\psi_{j}(x)\right] L_{j}(x) d F_{j}(x) \text { subject to } 0 \leq L_{j}(x) \leq x,
\end{aligned}
$$

where we denote:

$$
\begin{aligned}
& q_{j}(x)=E\left[u_{0}^{\prime}(A) \mid X_{j}=x\right] / E u_{0}^{\prime}(A) \text { and } \\
& r_{j}(x)=E\left[u_{j}^{\prime}\left(B_{j}\right) \mid X_{j}=x\right] / E u_{j}^{\prime}\left(B_{j}\right), j=1, \ldots, n .
\end{aligned}
$$

A characterization of solutions to this kind of optimization problems is given by a result known as the Neyman-Pearson lemma (see e.g. Lehmann (1959), and Golubin (2006) for an application to insurance problems). According to it, an admissible policy $I_{j}(x)$ is optimal in (8) if and only if $I_{j}(x)=0$ on $x$ : $\psi_{j}(x)-q_{j}(x)<0$ and $I_{j}(x)=x$ on $x: \psi_{j}(x)-q_{j}(x)>0$ up to a set in $[0, \infty)$ of zero $F_{j}$-measure. Applying the Neyman-Pearson lemma to problem (9) also, we have 


$$
I_{j}(x)=\left\{\begin{array}{l}
0, \text { for } x: r_{j}(x) \leq \psi_{j}(x) \leq q_{j}(x) \text { and } r_{j}(x)<q_{j}(x) \\
x, \text { for } x: r_{j}(x) \geq \psi_{j}(x) \geq q_{j}(x) \text { and } r_{j}(x)>q_{j}(x)
\end{array}\right.
$$

up to a set of zero $F_{j}$-measure. Remark that since $E \Psi_{j}=1$, from (10)-(11) we get

$$
\int_{0}^{\infty}\left[\psi_{j}(x)-q_{j}(x)\right] d F_{j}(x)=0=\int_{0}^{\infty}\left[r_{j}(x)-\psi_{j}(x)\right] d F_{j}(x) .
$$

Note also that the definitions (10)-(11) of $r_{j}$ and $q_{j}$ along with definitions of the capitals $B_{j}$ and $A$ (in the latter, recall, all $I_{s}\left(X_{s}\right), s \neq j$, are independent of $X_{j}$ ) allow for representing the functions as $q_{j}(x)=s_{j}\left(-I_{j}(x)\right)$ and $r_{j}(x)=v_{j}(-x+$ $\left.I_{j}(x)\right)$, where $s_{j}(\cdot)$ and $v_{j}(\cdot)$ are some decreasing functions.

Now prove that (12) implies $\psi_{j}(x) \equiv q_{j}(x)$ and $\psi_{j}(x) \equiv r_{j}(x)$ almost everywhere with respect to $F_{j}$-measure. Suppose at first that as $x$ increases from 0 we have $r_{j}(x) \leq \psi_{j}(x) \leq q_{j}(x)$ and $r_{j}(x)<q_{j}(x)$ (for definiteness, let $r_{j}(x)<\psi_{j}(x) \leq$ $\left.q_{j}(x)\right)$. Then, by (12), $I_{j}(x)$ remains equal to 0 , while $q_{j}(x)=s_{j}\left(-I_{j}(x)\right)$ is constant and $r_{j}(x)=v_{j}\left(-x+I_{j}(x)\right)$ is increasing. Such a pattern cannot be valid for all $x$ (with respect to $F_{j}$-measure) as (13) would be violated. Note in this connection that (12) excludes situations when $\psi_{j}(x)<\min \left\{r_{j}(x), q_{j}(x)\right\}$ or $\psi_{j}(x)>$ $\max \left\{r_{j}(x), q_{j}(x)\right\}$ as impossible for optimal $I_{j}$. Hence there must be encountered a set of arguments $x$ where

$$
r_{j}(x) \geq \psi_{j}(x) \geq q_{j}(x) \text { and } r_{j}(x)>q_{j}(x) .
$$

When $x$ reaches the set, $I_{j}(x)$ switches from 0 to $x$. This change of $I_{j}(x)$ implies a decrease of $r_{j}(x)$ and an increase of $q_{j}(x)$, which means a contradiction with attaining (14).

Suppose $r_{j}(x) \geq \psi_{j}(x) \geq q_{j}(x)$ and $r_{j}(x)>q_{j}(x)$ as $x$ increases from 0 . Similar to the reasonings above, we find in this case that at first $I_{j}(x)$ remains equal to $x$. After switching $I_{j}(x)$ to zero when inequalities $r_{j}(x) \leq \psi_{j}(x) \leq q_{j}(x)$ and $r_{j}(x)<q_{j}(x)$ become valid, there must be a decrease of $q_{j}(x)$ and an increase of $r_{j}(x)$. This contradicts the latter inequalities.

Following these reasonings, we come to that the only feasible case is $\psi_{j}(x) \equiv$ $q_{j}(x)$ and $\psi_{j}(x) \equiv r_{j}(x)$ up to a set of zero $F_{j}$-measure. From the definitions (10)(11) of $q_{j}(x)$ and $r_{j}(x)$ we get (5)-(6).

Clearly, if (5)-(6) hold then inequalities (7) are satisfied, converting into equalities. Hence, $I$ is optimal in (3).

Compare Theorem 1 and a characterization of equilibrium in the Borch's model of a reinsurance market (see Buhlmann $(1980,1984)): u_{i}^{\prime}\left(w_{i}-Z_{i}\right)=$ $\Phi E u_{i}^{\prime}\left(w_{i}-Z_{i}\right)$ a.s., where $Z_{i}$ is the "after exchange" risk of $i$-th agent in equilibrium. These optimality equations correspond in our case to $n$ pairs of 
equations (5)-(6) - recall that $2 n$ functions $\psi_{j}(x), I_{j}(x), j=1, \ldots, n$, are to be found in our setting. Also, the market price density $\Phi$ in the Borch's model is a function $\Phi=\Phi\left(X_{M}\right)$ of total initial risk $X_{M}=X_{1}+\ldots+X_{n}$ (as well as the optimal risk exchanges $\left.Z_{i}\right)$, while in (5)-(6) the $j$-th price density $\Psi_{j}=\psi_{j}\left(X_{j}\right)$ depends on the $j$-th insured's risk $X_{j}$ only.

Examine a degenerated case of risk-neutral insurer, i.e., $u_{0}(x)=x$. Since $u_{0}^{\prime}(x) \equiv 1$, from (5) we have $\Psi_{j}=1$ a.s. By (6), $I_{j}(x)=x$ for all $j$ and, thus, in equilibrium the only optimal policy is full coverage of the losses, the premiums $E\left[\Psi_{j} I_{j}\left(X_{j}\right)\right]$ coincide with the actuarial values $E X_{j}$.

Remark 1. Upon obtaining the equilibrium price functions $\psi_{j}(x) j=1, \ldots, n$, one can, in view of (4), put the market price density $\Phi=\prod_{j=1}^{n} \psi_{j}\left(X_{j}\right)$. Another and, perhaps, more natural way is to put

$$
\Phi=u_{0}^{\prime}(A) / E u_{0}^{\prime}(A),
$$

with the equilibrium indemnities $I_{j}\left(X_{j}\right)$ substituted into the final capital $A$. Indeed, from (5) it follows $E\left[\left\{u_{0}^{\prime}(A) / E u_{0}^{\prime}(A)\right\} \mid X_{j}\right]=\psi_{j}\left(X_{j}\right)$ a.s. for all $j$.

\section{Pareto optimality}

Consider a risk exchange in the insurance market in which now the premium $P_{j}$ paid by the $j$-th insured is not defined by the price density and indemnity function as in the previous sections, but is regarded as an independent variable chosen jointly with a policy $0 \leq I_{j}(x) \leq x$. An insurance contract is identified with a pair $(P, I)$, where $P=\left(P_{1}, \ldots, P_{n}\right)$ is a vector of premiums paid by the $n$ insureds, and $I=\left(I_{1}, \ldots, I_{n}\right)$ is a vector-function of policies.

Like the notation in section 2, we introduce the insurer's expected utility (of his final capital) $J_{0}[P, I] \stackrel{\text { def }}{=} E u_{0}\left(w_{0}+\sum_{s=1}^{n}\left(P_{s}-I_{s}\left(X_{s}\right)\right)\right)$ and the insured's expected utility $J_{j}[P, I] \stackrel{\text { def }}{=} E u_{j}\left(w_{j}-P_{j}-X_{j}+I_{j}\left(X_{j}\right)\right), j=1, \ldots, n$. By definition, a contract $(\hat{P}, \hat{I})$ is called Pareto-optimal if there is no other contract $(P, I)$ such that $J_{i}[P, I] \geq J_{i}[\hat{P}, \hat{I}]$ for $i=0, \ldots, n$, and at least one of the inequalities is strict. That is, under the contract any agent cannot improve his utility without worsening the utility of at least one other agent.

A method for obtaining a Pareto-optimal solution can be found in Gerber (1978) (see also Borch (1960) and Aase (2002)) and consists in maximization of a weighted sum of the agents' utilities: Fix a vector $k=\left(k_{0}, \ldots, k_{n}\right)$ such that $k>0$ component-wise and $\sum_{0}^{n} k_{i}=1$, then maximize the functional $\sum_{i=0}^{n} k_{i} J_{i}[P, I]$ over the set of insurance contracts $(P, I)$.

Show that in our case this procedure generates the set of all Pareto-optimal contracts. As is known (see, e.g., Gerber (1978) and Aase (2002)), if $(\hat{P}, \hat{I})$ is a Pareto-optimal solution then, due to concavity of all the functionals $J_{i}$, there exists a vector $k \geq 0, \sum_{0}^{n} k_{i}=1$ such that $(\hat{P}, \hat{I})$ is a maximizer in the 
problem above. Suppose $k_{0}=0$, then there is $k_{j}>0$ for some $j>0$. Since $J_{j}[\hat{P}, \hat{I}]=E u_{j}\left(w_{j}-\hat{P}_{j}-X_{j}+\hat{I}_{j}\left(X_{j}\right)\right)$, we can choose $P_{j}<\hat{P}_{j}$ that gives a greater value of the weighted sum of the utilities, which leads to a contradiction with optimality of $(\hat{P}, \hat{I})$. Suppose $k_{j}=0$ for some $j>0$. As $k_{0}>0$ and $J_{0}[\hat{P}, \hat{I}]=$ $E u_{0}\left(w_{0}+\sum_{s=1}^{n}\left(\hat{P}_{s}-\hat{I}_{s}\left(X_{s}\right)\right)\right)$, we can choose a contract with $P_{j}>\hat{P}_{j}$ at which the weighted sum has a greater value so that we come to a contradiction.

For convenience we set $\delta_{j}=k_{j} / k_{0}$ and rewrite the maximization problem above as

$$
\operatorname{maximize} J[P, I] \equiv J_{0}[P, I]+\sum_{j=1}^{n} \delta_{j} J_{j}[P, I],
$$

where $\delta_{j}>0$, and maximization is taken over $P \in R^{n}$ and $I=\left(I_{1}, \ldots, I_{n}\right): 0 \leq$ $I_{j}(x) \leq x$ for $j=1, \ldots, n$. As we have shown, the $n$-parameter family $\left\{\left(\hat{P}^{\delta}, \hat{I}^{\delta}\right)\right\}_{\delta>0}$ of maximizers in (15) consists of all Pareto-optimal contracts.

The problem of finding Pareto optimality conditions via solving (15) was studied in Golubin (2005). Below we reformulate the relevant theorems from the paper; it turns out that the theorems' statements are preserved, while the assumptions can now be weakened with respect to admitting the insureds' risks distributions $F_{j}$ with arbitrary supports ${ }^{1}$, not only with interval-shaped supports. The latter is achieved by employing the Neyman-Pearson lemma, instead of using the directional derivatives technique only.

The next theorem gives a characterization of the Pareto-optimal contract $(\hat{P}, \hat{I})=\left(\hat{P}^{\delta}, \hat{I}^{\delta}\right)$ (below we will omit the superscript $\delta$ for convenience) in the form of necessary and sufficient conditions for optimality in (15). Denote, respectively, by

$$
A=w_{0}+\sum_{s=1}^{n}\left(\hat{P}_{s}-\hat{I}_{s}\left(X_{s}\right)\right) \text { and } B_{j}=w_{j}-\hat{P}_{j}-X_{j}+\hat{I}_{j}\left(X_{j}\right)
$$

the final capitals of the insurer and $j$-th insured under a contract $(\hat{P}, \hat{I})$.

Theorem 2. A contract $(\hat{P}, \hat{I})$ solves (15) - i.e., it is Pareto-optimal - if and only if

$$
E\left[u_{0}^{\prime}(A) \mid X_{j}\right]=\delta_{j} u_{j}^{\prime}\left(B_{j}\right) \text { a.s. } j=1, \ldots, n .
$$

Proof. Let $(\hat{P}, \hat{I})$ be a maximizer in (15). Denote $\Delta P=P-\hat{P}$, where $P$ is an arbitrary vector in $R^{n}$, and let $\Delta(x)=\left(\Delta_{1}(x), \ldots, \Delta_{n}(x)\right)$ be a vector-function with $\Delta_{j}=I_{j}-\hat{I}_{j}$, where $I_{j}$ is an arbitrary policy, $j=1, \ldots, n$. For $\lambda \in[0,1]$ define $P_{\lambda}=\hat{P}+\lambda \Delta P$ and $I_{\lambda}=\hat{I}+\lambda \Delta$, and consider a function $J(\lambda) \stackrel{\text { def }}{=} J\left[P_{\lambda}, I_{\lambda}\right]$ (see (15)). Optimality of $\hat{I}$ means that $\lambda=0$ maximizes $J(\lambda)$ on the interval $[0,1]$. Due to

1 The support supp $F_{j}$ of a probability distribution $F_{j}$ is the least closed set $S \subseteq R$ such that $\left\{P\left\{X_{j} \in S\right\}\right.$ $=1\}$. For example, the support of a Poisson distribution is $\{0,1, \ldots\}$ and the support of an exponential distribution is $[0, \infty)$. 
concavity of all the functionals $J_{i}$, this is equivalent to $J^{\prime}(0) \leq 0$ for any $P$ and indemnity rule $I$, or

$$
\sum_{s=1}^{n} \Delta P_{s} E\left(u_{0}^{\prime}(A)-\delta_{s} u_{s}^{\prime}\left(B_{s}\right)\right)-\sum_{s=1}^{n} E\left\{\left[u_{0}^{\prime}(A)-\delta_{s} u_{s}^{\prime}\left(B_{s}\right)\right] \Delta_{s}\left(X_{s}\right)\right] \leq 0 .
$$

Setting $\Delta_{j}(x) \equiv 0$ for all $j$, by (18) we have

$$
E\left(u_{0}^{\prime}(A)-\delta_{j} u_{j}^{\prime}\left(B_{j}\right)\right)=0
$$

for $j=1, \ldots, n$ as (18) holds for any choice of $\Delta P$. Then fixing any $j$ and setting $\Delta_{s}(x) \equiv 0$ for all $s \neq j$, we obtain that (18) is equivalent to $E\left\{\left(\delta_{j} u_{j}^{\prime}\left(B_{j}\right)-\right.\right.$ $\left.\left.u_{0}^{\prime}(A)\right) \Delta_{j}\left(X_{j}\right)\right\} \leq 0, j=1, \ldots, n$. In other words, $\hat{I}_{j}$ is a solution to the problem

$$
\operatorname{maximize} \int_{0}^{\infty} \hat{V}_{j}(x) I_{j}(x) d F_{j}(x) \text { subject to } 0 \leq I_{j}(x) \leq x,
$$

where $\hat{V}_{j}(x) \stackrel{\text { def }}{=} E\left[\delta_{j} u_{j}^{\prime}\left(B_{j}\right)-u_{0}^{\prime}(A) \mid X_{j}=x\right]$. Analogous to the Theorem 1's proof, by the Neyman-Pearson lemma we have that a policy $\hat{I}_{j}$ solves $(20)$ if and only if

$$
\hat{I}_{j}(x)=\left\{\begin{array}{ll}
0, & \text { if } \hat{V}_{j}(x)<0 \\
x, & \text { if } \hat{V}_{j}(x)>0
\end{array} \text { up to a set of zero } F_{j}\right. \text {-measure. }
$$

First examine the case $\hat{V}_{j}(0)<0$. When $x$ increases from $x=0$, the function $\hat{V}(x)$ increases under $\hat{I}_{j}(x)=0$ (see the definition of $\hat{V}(x)$ along with (16)). By (19), there must be a point at which $\hat{I}_{j}(x)$ switches to $x$ as $\hat{V}_{j}(x)$ becomes positive. However, under this policy $\hat{V}_{j}(x)$ is a decreasing function as follows from concavity of $u_{0}(\cdot)$. Thus, we have a contradiction with the positiveness of $\hat{V}_{j}(x)$.

Suppose that $\hat{V}_{j}(0)>0$. Similar to the arguments above, at first $\hat{I}_{j}(x)$ is equal to $x$ with a decreasing function $\hat{V}_{j}(x)>0$. Then, after switching $\hat{I}_{j}(x)$ to zero when $\hat{V}_{j}(x)$ becomes negative, the function $\hat{V}_{j}(x)$ becomes increasing and we come to a contradiction. Thus, optimality of $\hat{I}_{j}$ in (20) (and, hence, in the initial problem (15)) means $\hat{V}_{j}(x) \equiv 0$ almost everywhere with respect to $F_{j}$-measure, that is, $E\left[u_{0}^{\prime}(A) \mid X_{j}\right]-\delta_{j} u_{j}^{\prime}\left(B_{j}\right)=0$ a.s. for all $j=1, \ldots, n$.

To continue the analysis, recall that the absolute risk aversion function of an agent is $r(x)=-\frac{u^{\prime \prime}(x)}{u^{\prime}(x)}$ and the reciprocal of it, $\rho(x)=1 / r(x)$, is the risk tolerance function. Consider (17) under given $X_{j}=x$ and formally differentiate the equation with respect to $x$. Expressing then $\delta_{j}$ from (17), we get a differential equation

$$
\hat{I}_{j}^{\prime}(x)=\rho_{0 j}(x) /\left[\rho_{0 j}(x)+\rho_{j}\left(B_{j}(x)\right)\right]
$$


with initial condition $\hat{I}_{j}(0)=0, j=1, \ldots, n$, where $B_{j}(x)=w_{j}-\hat{P}_{j}-x+\hat{I}_{j}(x)$ is the final insured's capital $B_{j}$ under $X_{j}=x$ (see (16)) and $\rho_{0 j}(x) \stackrel{\text { def }}{=}-E\left[u_{0}^{\prime}(A) \mid X_{j}=x\right] /$ $E\left[u_{0}^{\prime \prime}(A) \mid X_{j}=x\right]$. The introduced function $\rho_{0 j}(x)$ is the ratio of the two expectations conditioned by $X_{j}=x$, but not the expectation of the risk tolerance $\rho_{0}(A)=-u_{0}^{\prime}(A) / u_{0}^{\prime \prime}(A)$ under $X_{j}=x$. The form of $\rho_{0 j}(x)$ is thus generally affected by the risk distributions $F_{s}, s \neq j$. In the sequel it will be referred to as a risk tolerance ratio of the insurer with respect to $j$-th insured.

Corollary 1. A contract $(\hat{P}, \hat{I})$ is Pareto-optimal if and only if

$$
\begin{aligned}
& E\left[u_{0}^{\prime}(A) \mid X_{j}=0\right]=\delta_{j} u_{j}^{\prime}\left(w_{j}-\hat{P}_{j}\right) \text { for some } \delta_{j}>0 \text { and } \\
& \hat{I}_{j}^{\prime}\left(X_{j}\right)=\frac{\rho_{0 j}\left(X_{j}\right)}{\rho_{0 j}\left(X_{j}\right)+\rho_{j}\left(B_{j}\right)} \text { a.s. }
\end{aligned}
$$

with initial conditions $\hat{I}_{j}(0)=0, j=1, \ldots, n$.

The proof is given in Appendix.

Remark 2. In order to completely define an indemnity $I_{j}\left(X_{j}\right)$, it suffices to define the function $I_{j}(x)$ on the support supp $F_{j}$ of distribution $F_{j}$ only, not on the whole interval $[0, \infty)$. In this connection, the optimality of a solution to equations (22)-(23) stated in Corollary 1 is understood in the same sense as in Wyler (1990), where Pareto-optimal exchanges in the Borch's model were characterized:

(i) if premiums $\hat{P}_{j}$ and functions $\hat{I}_{j}(x)$ satisfy $(22)$ and solve $(21)$ on $[0, \infty)$ (or at least on $\left[0, T_{j}\right]$ if $\left.T_{j} \stackrel{\text { def }}{=} \sup \left\{\operatorname{supp} F_{j}\right\}<\infty\right)$ then $(\hat{P}, \hat{I})$ is optimal in (15); (ii) if $(\hat{P}, \hat{I})$ is optimal then there are functions $\bar{I}_{j}(x)$ satisfying $(22)$ and $(21)$ on $[0, \infty)$ (or on $\left[0, T_{j}\right]$ ) such that $\bar{I}_{j}(x) \equiv \hat{I}_{j}(x)$ on $\operatorname{supp} F_{j}, j=1, \ldots, n$. Roughly, this means that one can solve system of equations $(21)$ on, say, $[0, \infty)$ without regard to the shapes of $\operatorname{supp} F_{j}, j=1, \ldots, n$.

\section{EQUILIBRIUM IN TERMS OF RISK AVERSION}

Returning to the equilibrium concept, remark that an equilibrium $(\Psi, I)$ - according to the definition - gives the individually rational contract $(P, I)$, where the premium vector $P=E[\Psi I(X)]$ component-wise. By individual rationality we mean that the contract does not lessen the initial utilities, $J_{i}[P, I] \geq J_{i}[0,0]$, $i=0, \ldots, n$. Here the agents's initial utilities before making a contract are: $J_{0}[0,0]=u_{0}\left(w_{0}\right)$ for the insurer, and $J_{j}[0,0]=E u_{j}\left(w_{j}-X_{j}\right), j=1, \ldots, n$, for the insureds. Since $J_{0}[P, I]=E u_{0}\left(w_{0}+\sum_{s=1}^{n}\left(P_{s}-I_{s}\left(X_{s}\right)\right)\right) \geq J_{0}[0,0]=u_{0}\left(w_{0}\right)$ in equilibrium, by Jensen's inequality and monotonicity of $u_{0}(x)$ we have

$$
\sum_{j=1}^{n} E\left[\Phi I_{j}\left(X_{j}\right)\right] \geq \sum_{j=1}^{n} E I_{j}\left(X_{j}\right)
$$


- the summary premium exceeds the actuarial value of the summary risk taken by insurer.

In connection between Pareto optimality and equilibrium, it is worthwhile to note that from conditions (5)-(6) in Theorem 1 it follows that (17) in Theorem 2 is satisfied under a contract $(\hat{P}, \hat{I})$ equal to the equilibrium contract $(P, I)$ if we set $\delta_{j}=E u_{0}^{\prime}(A) / E u_{j}^{\prime}\left(B_{j}\right)$ for $j=1, \ldots, n$, i.e., the equilibrium contract is necessarily a Pareto optimum.

The next theorem shows how necessary and sufficient conditions for equilibrium in terms of risk aversion (tolerance) functions are obtained from Theorem 1 and Pareto optimality conditions in Corollary 1 . As before, $B_{j}(x)=w_{j}-$ $P_{j}-x+I_{j}(x)$ stands for the $j$-th insured's final capital under $X_{j}=x$.

Theorem 3. Collections $\Psi=\left(\Psi_{1}, \ldots, \Psi_{n}\right)$ and $I=\left(I_{1}, \ldots, I_{n}\right)$ constitute an equilibrium in the market if and only if

$$
\Psi_{j}=\frac{\exp \int_{0}^{X_{j}}\left[\rho_{0 j}(t)+\rho_{j}\left(B_{j}(t)\right)\right]^{-1} d t}{E\left\{\exp \int_{0}^{X_{j}}\left[\rho_{0 j}(t)+\rho_{j}\left(B_{j}(t)\right)\right]^{-1} d t\right\}} \text { a.s. }
$$

and policies $I_{j}$ satisfy

$$
I_{j}^{\prime}(x)=\frac{\rho_{0 j}(x)}{\rho_{0 j}(x)+\rho_{j}\left(B_{j}(x)\right)}
$$

with initial conditions $I_{j}(0)=0$ and premiums $P_{j}$ equal to $E\left[\Psi_{j} I_{j}\left(X_{j}\right)\right], j=1, \ldots, n$.

Proof. 1. Suppose $(\Psi, I)$ to be an equilibrium. Then, by Theorem 1, (5) and (6) hold. Hence condition (17) in Theorem 2 is satisfied under $\hat{I}_{j}=I_{j}, \hat{P}_{j}=$ $E\left[\Psi_{j} I_{j}\left(X_{j}\right)\right]$, and $\delta_{j}=E u_{0}^{\prime}(A) / E u_{j}^{\prime}\left(B_{j}\right)$. Then from Corollary 1 we get that the policies $I_{j}$ satisfy (25).

To prove (24), for given $X_{j}=x$ we can write (5) as

$$
E u_{0}^{\prime}\left(A_{j}(x)\right)=\psi_{j}(x) E u_{0}^{\prime}(A),
$$

where $A_{j}(x)$ is a stochastic-valued function $A_{j}(x)=w_{0}+H\left[I_{j}\left(X_{j}\right)\right]-I_{j}(x)+$ $\sum_{s \neq j}^{n}\left\{H\left[I_{s}\left(X_{s}\right)\right]-I_{s}\left(X_{s}\right)\right\}$. Formal differentiating (26) with respect to $x$ and then dividing $\psi_{j}^{\prime}(x)$ by $\psi_{j}(x)$ expressed from (26) give

$$
\psi_{j}^{\prime}(x) / \psi_{j}(x)=-I_{j}^{\prime}(x) E u_{0}^{\prime \prime}\left(A_{j}(x)\right) / E u_{0}^{\prime}\left(A_{j}(x)\right) .
$$

Inserting the expression for $I_{j}^{\prime}(x)$ given by (23), we obtain a differential equation

$$
\psi_{j}^{\prime}(x) / \psi_{j}(x)=1 /\left[\rho_{0 j}(x)+\rho_{j}\left(B_{j}(x)\right)\right] .
$$


Taking into account the norming condition $\int_{0}^{\infty} \psi_{j}(x) d F_{j}(x)=1$, a unique solution to this equation is

$$
\bar{\psi}_{j}(x)=\frac{\exp \int_{0}^{x}\left[\rho_{0 j}(t)+\rho_{j}\left(B_{j}(t)\right)\right]^{-1} d t}{E\left\{\exp \int_{0}^{X_{j}}\left[\rho_{0 j}(t)+\rho_{j}\left(B_{j}(t)\right)\right]^{-1} d t\right\}} .
$$

In the same way as in the Corollary 1's proof, it is shown that the equilibrium density function $\psi_{j}(x)$ we start from coincides with $\bar{\psi}_{j}(x)$ up to a set of zero $F_{j}$-measure. Hence, expression (24) for $\Psi_{j}=\psi_{j}\left(X_{j}\right)$ is valid.

2. Let (25) hold with $P_{j}=E\left[\Psi_{j} I_{j}\left(X_{j}\right)\right]$, and $\Psi_{j}$ is given by $(24), j=1, \ldots, n$. Define a function $\psi_{j}(x)$ by the right-hand side of (28). As was shown in the first part of the proof, $\psi_{j}(x)$ satisfies equation (27) and, hence, coincides with $E u_{0}^{\prime}\left(A_{j}(x)\right)$ up to a multiplier. This along with the norming condition $E \psi_{j}\left(X_{j}\right)=1$ yields

$$
\psi_{j}(x)=E u_{0}^{\prime}\left(A_{j}(x)\right) / E u_{0}^{\prime}(A) .
$$

On the other hand, defining $\delta_{j}$ by equations $E\left[u_{0}^{\prime}(A) \mid X_{j}=0\right]=\delta_{j} u_{j}^{\prime}\left(w_{j}-P_{j}\right)$, we have from (25) and Corollary 1 that the policies $I_{j}$ and premiums $P_{j}$ constitute a Pareto-optimal contract. Then Theorem 2 gives

$$
E u_{0}^{\prime}\left(A_{j}(x)\right)=\delta_{j} u_{j}^{\prime}\left(B_{j}(x)\right) \text {, whence } \delta_{j}=E u_{0}^{\prime}(A) / E u_{j}^{\prime}\left(B_{j}\right) .
$$

Coupling (29) and (30), we have $E\left[u_{0}^{\prime}(A) \mid X_{j}\right]=\Psi_{j} E u_{0}^{\prime}(A)$ and $u_{j}^{\prime}\left(B_{j}\right)=\Psi_{j}$ $E u_{j}^{\prime}\left(B_{j}\right)$ a.s. for $j=1, \ldots, n$. According to Theorem 1, it means that $(\Psi, I)$ is an equilibrium.

Theorem 3 states in particular that the policies in equilibrium (if exists) solve equations (25) (see also Remark 2) and, hence, they satisfy $0<I_{j}^{\prime}(x)<1$. Since $I_{j}(0)=0$, any $I_{j}$ is a coinsurance policy with no deductible. This is, of course, a direct consequence of the coinsurance form of Pareto-optimal policies as given by (23) in Corollary 1 .

The market price density for the reinsurance market model (see Buhlmann (1984)) is a function of total risk $X_{M}$

$$
\Phi=\int_{0}^{X_{M}} 1 / \rho(x) d x / E\left[\int_{0}^{X_{M}} 1 / \rho(x) d x\right] \text { with the "total risk tolerance" }
$$

$\rho(x)=\sum_{i} \rho_{i}\left(w_{i}-Z_{i}(x)\right)$ involving the risk tolerance functions $\rho_{i}$ of all the agents. In distinction to it, expression (24) for the $j$-th agent's price density reflects, in general, the presence of the other $n-1$ insureds in the market by dependence of the risk tolerance ratio $\rho_{0 j}(x)=-E\left[u_{0}^{\prime}(A) \mid X_{j}=x\right] / E\left[u_{0}^{\prime \prime}(A) \mid X_{j}=x\right]$ on distributions of $X_{s}$ for all $s \neq j$. Note that in the case $n=1$ (one insured only) this dependence disappears, and relations (24)-(25) becomes the same as equilibrium 
characterization in the Borch's model. For example, (25) converts into the differential equation presented in Aase (2002)

$$
I^{\prime}(x)=r_{1}\left(w_{1}-P-x+I(x)\right) /\left\{r_{0}\left(w_{0}+P-I(x)\right)+r_{1}\left(w_{1}-P-x+I(x)\right)\right\} .
$$

Example 1. Consider the problem of finding an equilibrium in the case of exponential utility functions of the agents, $u_{i}(x)=c_{i}^{-1}\left(1-\exp \left(-c_{i} x\right)\right), i=0, \ldots, n$. Without loss of generality we can assume the initial capitals $w_{i}=0$, because in this case $w_{i}$ can be excluded from both sides of optimality equations (5)-(6).

We start with determining the Pareto-optimal contracts $(\hat{P}, \hat{I})$. As shown in Golubin (2005), equation (23) gives, as $\rho_{0 j}(x) \equiv c_{i}^{-1}$ and $\rho_{j}(x) \equiv c_{j}^{-1}$, that

$$
\hat{I}_{j}(x)=\frac{c_{j}}{c_{0}+c_{j}} x, j=1, \ldots, n,
$$

are the only Pareto-optimal indemnity rule. Equation (22) results in

$$
\hat{P}_{j}=\frac{1}{c_{j}}\left\{-\ln \delta_{j}-a_{j}+d\left[\sum_{s=1}^{n} c_{s}^{-1}\left(\ln \delta_{s}+a_{s}\right)+a / c_{0}\right]\right\},
$$

where we introduce $d$ as defined by $d^{-1}=c_{0}^{-1}+\ldots c_{n}^{-1}$, and $a=\sum_{s=1}^{n} a_{s}$ with $a_{s}=\ln \left\{E \exp \left[X_{s} c_{0} c_{s} /\left(c_{0}+c_{s}\right)\right]\right\}<\infty$ by assumption. Thus the set of Pareto-optimal contracts $\left\{\left(\hat{P}^{\delta}, \hat{I}^{\delta}\right)\right\}_{\delta>0}$ consists of a single indemnity rule $\hat{I}$ and a range of premium vectors $\hat{P}=\hat{P}^{\delta}$ defined in (31). Summing $\hat{P}_{j}$ gives the summary premium

$$
\sum_{j=1}^{n} \hat{P}_{j}=\frac{d}{c_{0}} \sum_{j=1}^{n} c_{j}^{-1}\left(-\ln \delta_{j}+\sum_{s \neq j}^{n} a_{s}\right) .
$$

From (31) it is seen that the premium $\hat{P}_{j}$ depends on the distributions of the insureds losses $X_{s}$ through the quantities $a_{s}$. This dependence has a curious form: a coefficient at $a_{j}$ in (31) is $c_{j}^{-1}\left[-1+\left(c_{0}^{-1}+c_{j}^{-1}\right) d\right]<0$, while for all $s \neq j$ the coefficients at $a_{s}$ are positive. It looks like a "premium paradox" - if the $j$-th insured's risk is worsened by adding a positive constant, $X_{j}^{\prime}=X_{j}+b$, then the Pareto-optimal premium he pays decreases as $a_{j}^{\prime}>a_{j}$. The summary premium (see (32)) becomes greater, which is explained by increases in premiums $\hat{P}_{s}$ of all other insureds as follows from (31) if we consider the expressions for $\hat{P}_{s}$. An explanation of the effect seems the following. If the risk $X_{j}$ is worsened (i.e. increased with probability one), a "fair arbitrator" should worsen (i.e. decrease) the insured's weight $\delta_{j}$ so that to make the $j$-th insured pay more.

The equilibrium notion singles out a contract from the set of Pareto optima, playing in some sense the role of the "fair arbitrator". Expression (24) gives the $j$-th price density

$$
\Psi_{j}=\exp \left[X_{j} c_{0} c_{j} /\left(c_{0}+c_{j}\right)\right] / E \exp \left[X_{j} c_{0} c_{j} /\left(c_{0}+c_{j}\right)\right],
$$


from (25) we have the policy $I_{j}(x)=x c_{j} /\left(c_{0}+c_{j}\right), j=1, \ldots, n$. According to Theorem 3, the pair $(\Psi, I)$ is an equilibrium. The premium paid by $j$-th insured is

$$
P_{j}=E\left[\Psi_{j} I_{j}\right]=E\left[\exp \left(c_{0} I_{j}\right) I_{j}\right] / E\left[\exp \left(c_{0} I_{j}\right)\right],
$$

where we denote $I_{j}=I_{j}\left(X_{j}\right)$. Prices given by expressions of this kind are often related to the Esscher principle in actuarial mathematics. Buhlmann (1980) used the Esscher principle in pricing a risk newcoming into a reinsurance market. A discussion of some its properties can be found in Goovaerts et al. (1984). Particularly, the price value increases vs an increase in the coefficient $c_{0}$ and, hence, always dominates the actuarial value $E I_{j}$.

Returning to the "premium paradox", it is seen from (33) that for the worsened risk $X_{j}^{\prime}=X_{j}+b$ we have that $\Psi_{j}$ does not change and then the new premium $P_{j}^{\prime}=E\left[\Psi_{j} I_{j}\left(X_{j}^{\prime}\right)\right]$ quite naturally becomes greater than $P_{j}$.

Finally, we can also determine the market price density by use of (4) which yields

$$
\Phi=\prod_{j=1}^{n} \Psi_{j}=\exp \left[c_{0} \sum_{1}^{n} X_{j} c_{j} /\left(c_{0}+c_{j}\right)\right] / E \exp \left[c_{0} \sum_{1}^{n} X_{j} c_{j} /\left(c_{0}+c_{j}\right)\right] .
$$

If the insureds have identical risk aversion parameters, $c_{1}=\ldots=c_{n}$, the market price density can be rewritten as

$$
\Phi=\frac{\exp \left[\frac{c_{0} c_{1}}{c_{0}+c_{1}} X_{M}\right]}{E \exp \left[\frac{c_{0} c_{1}}{c_{0}+c_{1}} X_{M}\right]} \text {, where } X_{M}=\sum_{j=1}^{n} X_{j} .
$$

Compare the insurer's situation (his indemnity and premium received) with that in the Borch's model of reinsurance market where treaties among insureds are allowed. In both cases the initial risk portfolio of the $(n+1)$ agents is $\left(0, X_{1}, \ldots, X_{n}\right)$ with independent components. In our notation, the equilibrium risk exchange in the reinsurance market described, e.g., in Aase (2002) results in the $i$-th agent's risk $c_{i}^{-1} d X_{M}$ and the premium (i.e. the side payment he pays) $E\left[\exp \left(d X_{M}\right)\left(X_{i}-c_{i}^{-1} d X_{M}\right)\right] / E \exp \left(d X_{M}\right), i=0, \ldots, n$. Thus the insurer, i.e., the agent having number $i=0$ takes indemnity $\tilde{Y}=c_{0}^{-1} d X_{M}$ and receives the payment $\tilde{S}=$ $E\left[c_{0}^{-1} d X_{M} \exp \left(d X_{M}\right)\right] / E \exp \left(d X_{M}\right)=E\left[\tilde{Y} \exp \left(c_{0} \tilde{Y}\right)\right] / E \exp \left(c_{0} \tilde{Y}\right)$.

In our setting, the insurer's indemnity

$$
Y=\sum_{j=1}^{n} \frac{c_{j}}{c_{0}+c_{j}} X_{j}>\tilde{Y} \text { a.s. }
$$

The latter inequality follows from that $c_{j} /\left(c_{0}+c_{j}\right)=c_{0}^{-1} /\left(c_{j}^{-1}+c_{0}^{-1}\right)>c_{0}^{-1} / \sum_{0}^{n} c_{i}^{-1}$. The summary premium the insurer gets is $S=E[\Phi Y]=E\left[Y \exp \left(c_{0} Y\right)\right] / E \exp \left(c_{0} Y\right)$. To compare $\tilde{S}$ and $S$, we express them in the forms 


$$
\begin{aligned}
& \tilde{S}=c_{0}^{-1} \sum_{1}^{n} E\left[d X_{j} \exp \left(d X_{j}\right)\right] / E \exp \left(d X_{j}\right) \text { and } \\
& S=c_{0}^{-1} \sum_{1}^{n} E\left[m_{j} X_{j} \exp \left(m_{j} X_{j}\right)\right] / E \exp \left(m_{j} X_{j}\right),
\end{aligned}
$$

where $m_{j}=c_{0} c_{j}\left(c_{0}+c_{j}\right)^{-1}>d$. According to the above-mentioned monotonicity property of the Esscher principle, $E\left[X_{j} \exp \left(d X_{j}\right)\right] / E \exp \left(d X_{j}\right)<E\left[X_{j} \exp \left(m_{j} X_{j}\right)\right] /$ $E \exp \left(m_{j} X_{j}\right)$ for each $j$ as $d<m_{j}$ and, hence, $\tilde{S}<S$. We can conclude that in the reinsurance model, where insureds can exchange their risks among themselves, the insurer is more discrete in the sense that in equilibrium he takes a less indemnity (with probability one), receiving a less premium.

\section{EXISTENCE OF EQUILIBRIUM}

In Example 1 we have found in an explicit form the only pair $(\Psi, I)$ that satisfies Theorem 3 and, thus, is a unique equilibrium. For a general case it is desirable to have a set of conditions that guarantee the existence of an equilibrium. The problem of equilibrium existence in the reinsurance market model was studied by Buhlmann (1984) and Aase (1993). In the latter paper, based on results in Mas-Colell (1986), a set of relatively weak sufficient conditions for the existence was obtained. Buhlmann investigated the same problem in the case of bounded risks by using a parametrization of Pareto-optimal risk exchanges through their initial receipts (or, in other words, side payments).

In this section we focus on finite-dimensional case, supposing in addition to the assumptions in section 2 that each risk $X_{j}$ is a discrete stochastic variable with the finite set of possible values $x_{j}=\left(x_{j 1}, \ldots, x_{j S_{j}}\right)$. An indemnity function $I_{j}(x)$ is identified with a vector $I_{j} \in R^{S_{j}}$ satisfying $0 \leq I_{j} \leq x_{j}$ component-wise. The premium $P_{j}=E\left[\Psi_{j} I_{j}\left(X_{j}\right)\right]$ is thus a scalar product with respect to the probabilistic measure,

$$
P_{j}=\left\langle\psi_{j}, I_{j}>=\sum_{k=1}^{S_{j}} \psi_{j, k} I_{j k} P\left\{X_{j}=x_{j k}\right\}\right.
$$

where the vector $\psi_{j}=\left(\psi_{j 1}, \ldots, \psi_{j S_{j}}\right)$ corresponds to $j$-th price density function $\psi_{j}(x)$ and satisfies the inequality $\psi_{j} \geq 0$ and the norming condition $\left\langle\psi_{j}, \mathbf{1}\right\rangle=1$.

In order to establish the equilibrium existence, we employ Theorem 1 and prove in our case the solvability of equations (5)-(6) that can be written as

$$
\begin{array}{ll}
r_{j}\left[\Psi, I, X_{j}\right]-\Psi_{j}=0 & \text { a.s. } \\
\Psi_{j}-q_{j}\left[\Psi, I, X_{j}\right]=0 & \text { a.s. }
\end{array}
$$

where, following the notation of Theorem $1, r_{j}[\Psi, I, x]=E\left[u_{j}^{\prime}\left(B_{j}\right) \mid X_{j}=x\right] /$ $E u_{j}^{\prime}\left(B_{j}\right)$ and $q_{j}[\Psi, I, x]=E\left[u_{0}^{\prime}(A) \mid X_{j}=x\right] / E u_{0}^{\prime}(A), A$ and $B_{j}$ stand for the final 
capitals of the insurer and insureds respectively, $j=1, \ldots, n$. The next lemma allows for reducing the question on solvability of (34)-(35) (with respect to $(\Psi, I))$ to solvability of definite inequalities.

Lemma 1. Let an admissible $\left(\Psi^{*}, I^{*}\right)$ be such that

$$
\begin{aligned}
& E\left\{\left[r_{j}\left[\Psi^{*}, I^{*}, X_{j}\right]-\Psi_{j}^{*}\right]\left[\Psi_{j}-\Psi_{j}^{*}\right]\right\} \leq 0 \\
& E\left\{\left[\Psi_{j}^{*}-q_{j}\left[\Psi^{*}, I^{*}, X_{j}\right]\right]\left[I_{j}\left(X_{j}\right)-I_{j}^{*}\left(X_{j}\right)\right]\right\} \leq 0
\end{aligned}
$$

for any admissible $\Psi_{j}$ and $I_{j}\left(X_{j}\right), j=1, \ldots, n$. Then $\left(\Psi^{*}, I^{*}\right)$ solves $(34)-(35)$.

The proof is given in Appendix.

Now we prove the existence of a solution $\left(\Psi^{*}, I^{*}\right)$ to (36)-(37). For this purpose we represent the inequalities in the form of a finite-dimensional variational inequality problem (see Harker and Pang (1990) for a review of this kind of problems). Since in the considered finite-dimensional case the expectations can be rewritten as the scalar products with the use of the above-introduced vectors $\psi_{j} \in R^{S_{j}}$ and $I_{j} \in R^{S_{j}}$, (36)-(37) take the form

$$
\begin{aligned}
& <g_{j}\left(\psi^{*}, I^{*}\right), \psi_{j}-\psi_{j}^{*}>\leq 0 \\
& <f_{j}\left(\psi^{*}, I^{*}\right), I_{j}-I_{j}^{*}>\leq 0
\end{aligned}
$$

for $j=1, \ldots, n$, where the $S_{j}$-dimensional vector-functions $g_{j}$ and $f_{j}$ correspond to the functions in the left-hand sides of (36) and (37) respectively. The set of admissible $(\psi, I)$ is

$$
\begin{aligned}
M=K \times L \stackrel{\text { def }}{=}\left\{\psi \in \prod_{j=1}^{n} R^{S_{j}}: \psi_{j} \geq\right. & \left.0,<\psi_{j}, \mathbf{1}>=1, j=1, \ldots, n\right\} \\
& \times\left\{I \in \prod_{j=1}^{n} R^{S_{j}}: 0 \leq I_{j} \leq x_{j}, j=1, \ldots, n\right\} .
\end{aligned}
$$

The fact that $M$ is the full Cartesian product of the individual sets $\left\{\psi_{j}\right\}$ and $\left\{I_{j}\right\}$ makes the system (38)-(39) of $2 n$ inequalities equivalent to the summary inequality

$$
\sum_{j=1}^{n}<g_{j}\left(\psi^{*}, I^{*}\right), \psi_{j}-\psi_{j}^{*}>+\sum_{j=1}^{n}<f_{j}\left(\psi^{*}, I^{*}\right), I_{j}-I_{j}^{*}>\leq 0 \text { for all }(\psi, I) \in M .
$$

The latter is related to the variational inequality problems and, by Theorem 3.1 in Harker and Pang (1990, p. 170), this inequality has a solution $\left(\psi^{*}, I^{*}\right)$ since all the functions $g_{j}(\psi, I)$ and $f_{j}(\psi, I)$ are continuous on the compact convex set $M$. Thus, according to Lemma 1, the system (34)-(35) is solvable and, by Theorem 1, an equilibrium exists in the insurance market model. 
Remark 3. Concerning a generalization of the equilibrium existence theorem to the infinite-dimensional case, the following directions seem worth attention: Under an assumption of boundedness of insureds' risks, $X_{j} \leq C$ a.s., one might try to use weak compactness of a set of bounded stochastic variables instead of the compactness property assumed in the above-mentioned theorem on solvability of variational inequality in Harker and Pang (1990, p. 170). Another way is to adjust the $L^{2}$-theory technique used in Aase (1993) to the case of the insurance market model.

\section{ACKNOWLEDGMENT}

The author wishes to thank anonymous referees for many helpful comments.

\section{APPENDIX}

The proof of Corollary 1 .

The way of the proof we use partially follows the reasonings in Wyler (1990, pp. 25-27). Assume, without loss of generality (see Remark 2), that the domains of all $\rho_{0 j}(x)$ and $\rho_{j}\left(B_{j}(x)\right)$ in $(23)$ are $[0, \infty)$.

(i) Let $\hat{P}$ and $\hat{I}(x)$ satisfy (22) and solve (21) on [0, $)$. Define differentiable functions on $[0, \infty): g_{j}(x)=E u_{0}^{\prime}\left(A_{j}(x)\right)-\delta_{j} u_{j}^{\prime}\left(B_{j}(x)\right)$, where $A_{j}(x) \stackrel{\text { def }}{=} w_{0}+\hat{P}_{j}-\hat{I}_{j}(x)+$ $\sum_{s \neq j}^{n}\left(\widehat{P}_{s}-\hat{I}_{s}\left(X_{s}\right)\right)$ and $B_{j}(x) \stackrel{\text { def }}{=} w_{j}-\hat{P}_{j}-x+\hat{I}_{j}(x), j=1, \ldots, n$. The derivative of $g_{j}(x)$ is $g_{j}^{\prime}(x)=-E u_{0}^{\prime \prime}\left(A_{j}(x)\right) \hat{I}_{j}^{\prime}(x)-\delta_{j} u_{j}^{\prime \prime}\left(B_{j}(x)\right)\left[\hat{I}_{j}^{\prime}(x)-1\right]$. Inserting the expression for $\hat{I}_{j}^{\prime}(x)$ given by $(21)$, we obtain

$$
\begin{aligned}
& g_{j}^{\prime}(x)=g_{j}(x) /\left[\rho_{0 j}(x)+\rho_{j}\left(B_{j}(x)\right)\right] \text { and, hence, } \\
& g_{j}(x)=C_{j} \exp \left(\int_{0}^{x}\left[\rho_{0, j}(t)+\rho_{j}\left(B_{j}(t)\right)\right]^{-1} d t\right) .
\end{aligned}
$$

From (22) we get $g_{j}(0)=0$, therefore $C_{j}=0$ and $g_{j}(x) \equiv 0$ for all $x \in[0, \infty)$ and $j=1, \ldots, n$. Because condition (17) in Theorem 2 evidently holds, $(\hat{P}, \hat{I})$ is optimal in (15).

(ii) Let $(\hat{P}, \hat{I})$ be a solution to (15). Choose any $j$ and fix $\hat{P}$ and $\hat{I}_{s}$ for $s \neq j$. Considering (21) as an equation on $[0, \infty)$, by a standard theorem on differential equations we have that $(21)$ has a unique solution $\bar{I}_{j}(x)$. As was shown in part (i), $\bar{I}_{j}(x)$ solves equation (17) on $[0, \infty)$ :

$$
E u_{0}^{\prime}\left(A_{j}(x)\right)=\delta_{j} u_{j}^{\prime}\left(B_{j}(x)\right) .
$$


The only solution of this equation (see the definitions of $A_{j}(x)$ and $B_{j}(x)$ presented above) can be written as $\bar{I}_{j}(x)=f_{j}^{-1}\left(w_{j}-\hat{P}_{j}-x\right)$, where $f_{j}^{-1}$ is the inverse of the function

$$
f_{j}(z)=\left(u_{j}^{\prime}\right)^{-1}\left(E u_{0}^{\prime}\left(w_{0}-z+\widehat{P}_{j}+\sum_{s \neq j}^{n}\left(\widehat{P}_{s}-\hat{I}_{s}\left(X_{s}\right)\right)\right) / \delta_{j}\right)-z .
$$

Note that $f_{j}^{-1}$ exists because $u_{0}^{\prime}(\cdot)$ and $u_{j}^{\prime}(\cdot)$ are decreasing functions. On the other hand, the optimality of $(\hat{P}, \hat{I})$ means, by Theorem 2 , that $\hat{I}_{j}(x)$ solves (40) for $x \in \operatorname{supp} F_{j}$. Uniqueness of the solution to (40) gives $\bar{I}_{j}(x) \equiv \hat{I}_{j}(x)$ on supp $F_{j}$. Repeating these arguments for each $j=1, \ldots, n$ completes the proof.

The proof of Lemma 1.

(i) Let (36) be satisfied, which means that $\psi_{j}^{*}(x)$ is a maximizer in the problem

$$
\max _{\psi_{j}} \int_{0}^{\infty} G_{j}(x) \psi_{j}(x) d F_{j}(x)
$$

where we denote $G_{j}(x)=r_{j}\left[\Psi^{*}, I^{*}, x\right]-\psi_{j}^{*}(x)$. Then all positive values of $\psi_{j}^{*}(x)$ correspond to the set $A^{*}$ of arguments $x$ at which $G_{j}(x)$ attains its maximum on supp $F_{j}$.

Suppose the contrary to the lemma's statement: $G_{j}(x) \not \equiv 0$ on supp $F_{j}$. By construction, $\int_{0}^{\infty} G_{j}(x) d F_{j}(x)=0$ hence $G_{j}(x)$ must change the sign and, in particular, its maximal value attained on $A^{*}$ is positive. However, $r_{j}\left[\Psi^{*}, I^{*}, x\right]>0$ for all $x$ and $\psi_{j}^{*}(x)=0$ for all $x \notin A^{*}$ so that $G_{j}(x)=r_{j}\left[\Psi^{*}, I^{*}, x\right]-\psi_{j}^{*}(x)>0$ everywhere with respect to $F_{j}$-measure. The obtained contradiction proves the required result: $G_{j}(x)=0$ for all $x \in \operatorname{supp} F_{j}$.

(ii) Let (37) be satisfied, that is, $I_{j}^{*}$ maximizes the integral

$$
\int_{0}^{\infty}\left(\psi_{j}^{*}(x)-q_{j}\left[\Psi^{*}, I^{*}, x\right]\right) I_{j}(x) d F_{j}(x) \text { subject to } 0 \leq I_{j}(x) \leq x .
$$

This problem coincides up to the notation with problem (8) considered in Theorem 1. Then, repeating the arguments in the Theorem 1's proof and observing that $r_{j}\left[\Psi^{*}, I^{*}, x\right] \equiv \psi_{j}^{*}(x)$ on supp $F_{j}$, we get equality (35).

\section{REFERENCES}

AASE, K.K. (1993) Equilibrium in a Reinsurance Syndicate; Existence, Uniqueness and Characterization. ASTIN Bulletin 22, 185-211.

Aase, K.K. (2002) Perspectives of Risk Sharing. Scand. Actuarial J. 2, 73-128.

BorCH, K. (1960) Reciprocal Reinsurance Treaties. ASTIN Bulletin 1, 171-191.

Borch, K. (1962) Equilibrium in a Reinsurance Market. Econometrica 30, 424-444.

Borch, K. (1990) Economics of Insurance. Edited by Aase K.K. and Sandmo A., Amsterdam, North-Holland. 
Bühlmann, H. (1980) An Economic Premium Principle. ASTIN Bulletin 11, 52-60.

Bühlmann, H. (1984) The General Economic Premium Principle. ASTIN Bulletin 14, 13-21.

Gerber, H.U. (1978) Pareto-optimal Risk Exchanges and Related Decision Problems. ASTIN Bulletin 10, 25-33.

Golubin, A.Y. (2005) Pareto-optimal Contracts in an Insurance Market. ASTIN Bulletin 35(2), 363-378.

Golubin, A.Y. (2006) Pareto-optimal Insurance Policies in the Models with a Premium Based on the Actuarial Value. Journal of Risk and Insurance 73, 469-487.

Goovaerts, M.J., De Vylder, F. and Haezendonck, J. (1984) Insurance Premiums. Theory and Applications. Amsterdam, North-Holland.

HARKER, P.T. and PANG, J.S. (1990) Finite-dimensional Variational Inequality and Nonlinear Complementarity Problems: A Survey of Theory, Algorithms and Applications. Mathematical Programming 48, 161-220.

Lehmann, E.L. (1959) Testing Statistical Hypotheses. Wiley and Sons, New York.

Lemaire, J. (1990) Borch's Theorem. In: Louberge, H. (ed.) Risk, Information and Insurance. Essays in the Memory of Karl H. Borch, Kluwer Academic Publishers, Boston, London, 15-37.

Mas-Collel, A. (1986) The Price Equilibrium Existence Problem in Topological Vector Lattices. Econometrica 54, 1039-1054.

Tucker, H.G. (1967) A Graduate Course in Probability. Academic Press, New York.

WyLER, E. (1990) Pareto Optimal Risk Exchanges and a System of Differential Equations: A Duality Theorem. ASTIN Bulletin 20, 23-32.

\section{Golubin AleXey Y.}

Russian Academy of Sciences

Design Information Technologies Center,

Marshala Zhukova Street, 30-A

Odintsovo

Moscow region, 143000

Russia

E-Mail: io@miem.edu.ru info@ditc.ras.ru 